\title{
Threshold-Based Data Exclusion Approach for Energy-Efficient Federated Edge Learning
}

\author{
Abdullatif Albaseer, Mohamed Abdallah, Ala Al-Fuqaha, and Aiman Erbad \\ Division of Information and Computing Technology, College of Science and Engineering, \\ Hamad Bin Khalifa University, Doha, Qatar \\ \{amalbaseer, moabdallah, aalfuqaha, AErbad\}@hbku.edu.qa
}

\begin{abstract}
Federated edge learning (FEEL) is a promising distributed learning technique for next-generation wireless networks. FEEL preserves the user's privacy, reduces the communication costs, and exploits the unprecedented capabilities of edge devices to train a shared global model by leveraging a massive amount of data generated at the network edge. However, FEEL might significantly shorten energy-constrained participating devices' lifetime due to the power consumed during the model training round. This paper proposes a novel approach that endeavors to minimize computation and communication energy consumption during FEEL rounds to address this issue. First, we introduce a modified local training algorithm that intelligently selects only the samples that enhance the model's quality based on a predetermined threshold probability. Then, the problem is formulated as joint energy minimization and resource allocation optimization problem to obtain the optimal local computation time and the optimal transmission time that minimize the total energy consumption considering the worker's energy budget, available bandwidth, channel states, beamforming, and local CPU speed. After that, we introduce a tractable solution to the formulated problem that ensures the robustness of FEEL. Our simulation results show that our solution substantially outperforms the baseline FEEL algorithm as it reduces the local consumed energy by up to $79 \%$.
\end{abstract}

Index Terms-Federated Edge Learning, Convergence time, Resource allocation, Energy consumption, Edge Intelligence.

\section{INTRODUCTION}

The rapid advancement in fifth-generation (5G) cellular networks and the Internet of Things (IoT) have brought a sharp rise in the volume of data generated by end devices (e.g., smartphones, IoT devices, and smart sensors) and cellular base stations (BSs) at the wireless network edge. According to recent research reports, the increase is projected to be in the order of billions of gigabytes per day by 2022 [1]. This data can bring valuable artificial intelligence (AI) services to end-users by leveraging deep learning [2] and mobile edge computing techniques [3], which have been evolving and converging rapidly in recent years under the umbrella of mobile edge learning. It is envisioned that mobile edge learning will be a core technology for sixth-generation (6G) cellular networks enabling new applications, such as virtual reality and augmented reality; therefore, actualizing the vision of network intelligence [4], [5]. However, transferring large amounts of data to a central unit has become very difficult because of the networks' constraints, scalability issues, limited bandwidth, and most importantly, users' privacy.

Recently, federated edge learning (FEEL) has shown great potential on the network edge to process data collaboratively among end-users while maintaining privacy since data remains on client devices and only local models are shared [6]. Also, FEEL significantly reduces the network traffic load, as users only need to share the models' parameters, which are fixed in size and structure across users.

In FEEL, the convergence of the learning algorithm may not be guaranteed. The algorithm's convergence rate determines the number of training rounds required to reach the desired training accuracy. The model update tasks in each training round depend on the computation and communications latencies and the energy budget existing at edge workers. Therefore, it is clear that training the local models involves computation and communication costs and energy constraints closely interlinked. Hence, a scalable FEEL system that guarantees application performance must consider the learning algorithms, the communications system, and the edge devices' energy consumption aspects. These aspects impose new challenges that must be considered in designing an efficient FEEL [3].

This paper addresses these issues by proposing a novel scheme for allocating computation and communication resources to support energy-efficient FEEL systems. We study FEEL using a realistic wireless network where the workers are connected to a radio-frequency (RF) base station (BS). Each worker trains its local model using its local data and sends the model parameters to the BS, which aggregates all local parameters to form a global model that is broadcast to all available workers for further updates. Since the BS is resource-constrained, only a subset of available workers is selected every round to perform the updates. Furthermore, the workers are energy-constrained with finite batteries. Thus, the resources must be allocated to workers that meet all the required constraints. The key contributions of this work can be summarized as follows:

- We utilize a system's approach that jointly considers the learning algorithms as well as the available computation and communication resources to design an energyefficient FEEL training algorithm. The proposed algorithm leverages the global model to filter the local data samples and then intelligently selects only the local training samples that improve the model's performance.

- We formulate a joint energy minimization and resource allocation problem for FEEL. This problem is then solved as a sub-problem using a local heuristic that filters the data samples and using the Golden-Section search method to optimize the communication and computation energy.

- We carry out extensive simulations using realistic feder- 
ated datasets to assess the proposed solutions' efficacy. Our experiments demonstrate that the proposed technique can reduce the local energy consumption by up to $79 \%$ compared to the baseline FEEL algorithm while achieving similar accuracy. To the best of our knowledge, this work is the first that introduces intelligent sample exclusion during FEEL rounds.

The rest of this paper is structured as follows: we review recent related works in Section III Then, we introduce the system model, learning model, and computation and communication models in Section IIII The problem statement is formulated in Section IV. Experimental results are presented in Section VI Finally, Section VII concludes this work and provides directions for future extensions.

\section{RELATED WORK}

Communication and energy-constrained FEEL has been investigated by several researchers in the recent literature [7][13]. The authors in [7] and [8] proposed to compress the model parameters exchanged between edge servers and devices in order to reduce the communication cost and allow more users to join the training rounds. Another method proposed by Wang et al. [9] to optimize the number of global rounds as well as the number of local updates and minimize the value of the loss function and increase the accuracy taken into consideration the communication limitation of the wireless medium. Nishio and Yonetani [11] addressed worker selection in heterogeneous settings and proposed an approach to select the workers that provide less computation and communication time. However, they did not consider the effects of data size on the convergence rate.

Furthermore, to address the transmission latency, the Broadband Analog Aggregation (BAA) scheme has been proposed in [10] to reduce the transmission time between edge devices and the orchestrator server, by utilizing the superposition property of wireless channels via over-the-air computation (AirComp) [12]. AirComp advocates that concurrent transmissions can diminish multiple access latency by a factor equivalent to the number of devices (e.g., 100 times for 100 devices). This is a promising solution for fast edge learning; however, the required analog modulation makes it challenging to deploy this multi-access scheme, i.e., BAA, in modern wireless systems (digital infrastructure). To tackle this challenge, Zhu et al. [14] proposed a digital aggregation method over multi-access channels, coined as One-Bit Broadband Digital Aggregation (OBDA), which represents the first attempt to implement BAA using digital modulation. Specifically, OBDA integrates the digital modulation scheme (e.g., quadrature amplitude modulation or QAM) and the state-of-the-art onebit quantizer [14]. Focusing on energy-constraints, Wang et al. [16] considered the problem of energy-efficient communication and local computation resource allocation over wireless channels. They formulated an optimization problem to minimize the completion time, energy consumed during the local computation, and transmission energy. Furthermore, the authors in [5] proposed an approach to minimize the total energy consumption across all workers during predefined

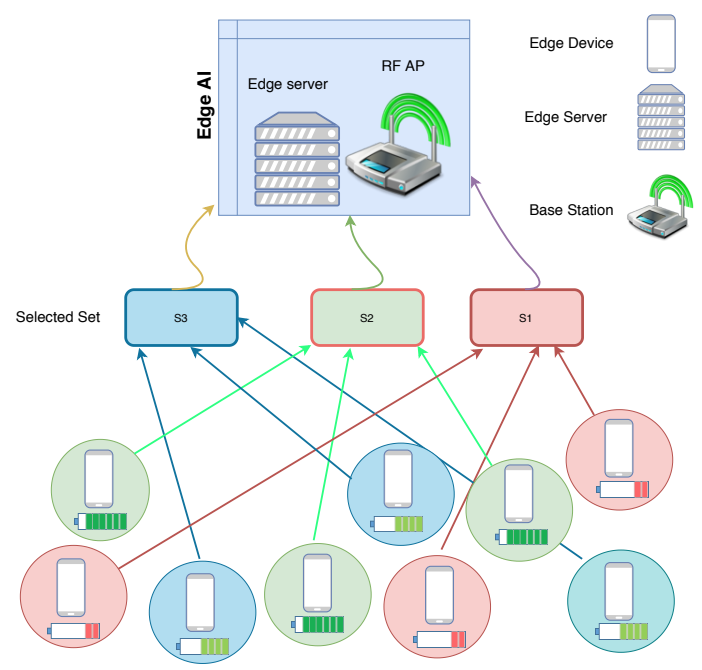

Fig. 1: The system model when a set of edge devices are connected to RF BS.

training time. They also formulated and solved the resulting optimization problem using numerical methods.

Despite such research efforts, there is a lack in designing an energy-efficient FEEL system, as illustrated in Table $\mathrm{I}$ considering optimizing the learning algorithm and the available communication computation edge network resources. This gap motivates us to propose a novel energy-efficient FEEL algorithm.

\section{SYSTEM MOdEL}

As depicted in Fig. 1. the system model used in this work consists of a set of edge devices $\mathcal{K}$ connected to a BS with $\mathrm{M}$-antennas that coordinates the workers to train a general global model to be used for future inference. Each worker $k \in \mathcal{K}$ has its own data $\mathcal{D}_{k}$ that is used to train its local model and send the update $\theta_{k}$ back to the server, where $\mathcal{D}_{k}=\left\{x_{k, d} \in \mathbb{R}^{d}, y_{k, d} \in \mathbb{R}\right\}$, and $\left|\mathcal{D}_{k}\right|$ is the number of local data samples. $x_{k, d}$ is the $d$-dimensional input data vector at the $k$-th worker, and $y_{k, d}$ is the corresponding label associated with $x_{k, d}$. In return, the server collects all the workers' updates and averages them to form a new global model. In the beginning, the server selects a subset of workers $S$ and then sends random initialization parameters $\theta_{0}$ so that the chosen workers can work accordingly. The selected workers use the received global model as reference when training local models to control the global and local models' divergence. After that, the server sends the updated model parameters $\theta_{r}$, which results from averaging the whole updates at the $r$-th round. To involve a specific worker in the training rounds, energy budget, computation, and communication capabilities are considered to establish a robust FEEL system that avoids losing selected worker updates due to insufficient energy or waiting a long time for stragglers.

\section{A. FEEL model}

The local loss function captures the error of the model on the local dataset $\left\{x_{k, d}, y_{k, d}\right\}$ for the $k$-th worker at the $r$-th 
TABLE I: RELATIONSHIP BETWEEN OUR WORK AND THE RECENT LITERATURE

\begin{tabular}{|c|l|l|l|l|l|}
\hline Ref & $\begin{array}{l}\text { Completion } \\
\text { Time }\end{array}$ & $\begin{array}{l}\text { Synchronize } \\
\text { the updates }\end{array}$ & $\begin{array}{l}\text { Devices } \\
\text { Heterogeneity }\end{array}$ & $\begin{array}{l}\text { Energy Bud- } \\
\text { get }\end{array}$ & $\begin{array}{l}\text { Training Algo- } \\
\text { rithm }\end{array}$ \\
\hline$[\overline{5}]$ & $\checkmark$ & X & $\checkmark$ & X & X \\
\hline$[15]$ & $\checkmark$ & $\times$ & $\checkmark$ & $X$ & $\times$ \\
\hline$[\overline{16}]$ & $\checkmark$ & $\times$ & $\checkmark$ & $X$ & $\times$ \\
\hline Our work & $\checkmark$ & $\checkmark$ & $\checkmark$ & $\checkmark$ \\
\hline
\end{tabular}

round, and it is defined as follows:

$$
F_{k}^{r}(\theta) \triangleq \frac{1}{\left|\mathcal{D}_{k}\right|} \sum_{s \in \mathcal{D}_{k}} f_{s}(\theta) .
$$

where $f_{s}$ captures the error of each local data sample. The total data across the edge network can be defined as follows: $D \triangleq \sum_{k=1}^{K}\left|\mathcal{D}_{k}\right|$, and the weight of the local data samples at the $k$-th worker $\delta_{k}$ can be defined as follows: $\delta_{k}=\frac{\left|\mathcal{D}_{k}\right|}{D}$.

To train its local model, the $k$-th worker runs its local solver, such as stochastic gradient descent (SGD), locally to minimize the loss function defined in Eq. (1) for several local epochs denoted by $\varepsilon$. Specifically, the local model parameters $\theta_{k}$ are updated as follows:

$$
\theta_{k}(n)=\theta_{k}(n-1)-\eta \nabla F_{k}^{r}\left(\theta_{k}(n)\right)
$$

where $n=1,2, \ldots, \varepsilon$ is the number of local updates performed by the $k$-th worker and $\eta$ is the step size (i.e., learning rate) at each round, $\theta_{k}(0)$ denotes the initial global parameters received from the server and $\theta_{k}(\varepsilon)$ denotes the last local parameters update the $k$-th worker sends back to the server after $\varepsilon$ rounds. For global loss function, after uploading all local model updates computed using (1) and (2), the weighted global loss function across workers at the $r$-th round is computed as:

$$
F_{r}(\theta) \triangleq \sum_{k=1}^{K} \delta_{k} F_{k}^{r}(\theta) .
$$

Accordingly, the global model parameters are computed as follow:

$$
\theta_{\mathbf{r}}=\sum_{k=1}^{K} \delta_{k} \theta_{k}
$$

$F_{r}(\theta)$ and $\theta_{\mathbf{r}}$ are sent to all selected workers to be used as a reference in the $(r+1)$-th round when updating the model parameters. Thus, the aim is to find $\theta^{*}$ so as to minimize $F(\theta)$

$$
\theta^{*} \triangleq \arg \min F(\theta) \text {. }
$$

\section{B. Local Computation model}

As stated before, the $k$-th worker holds $\left|D_{k}\right|$ data samples. To train its local model, the $k$-th worker splits the data $D_{k}$ into batches of size $b$ and trains its model for a number of epochs $\varepsilon$. Thus, the local computation time $T_{k}^{c m p}$ can be defined as:

$$
T_{k}^{c m p}=\varepsilon \frac{\left|D_{k}\right| \Phi}{f_{k}^{c m p}}
$$

where $f_{k}^{\mathrm{cmp}}$ denotes the local CPU frequency, and $\Phi$ denotes the number of cycles required to process one sample. Namely, the server sets a deadline $\mathbf{T}$ to synchronize the updates and avoid long waiting times especially for stragglers (i.e., the devices with low battery, low CPU speed, and bad channels). Thus, the $k$-th worker has to accomplish its computation and communication phases within $\mathbf{T}$ to ensure the update synchronization. Accordingly, the local computation time should satisfy this condition:

$$
T_{k}^{\mathrm{cmp}}=\mathbf{T}-T_{k}^{\mathrm{up}}
$$

where $T_{k}^{\text {up }}$ denotes the uploading time of the update to the server.

\section{Local Energy consumption model:}

From (6) the corresponding local energy consumption for every $k$-th worker due to local model training is defined as:

$$
E_{k}^{c m p}=\frac{\alpha_{k}}{2}\left(f_{k}^{\mathrm{cmp}}\right)^{3} T_{k}^{c m p}
$$

where $\frac{\alpha_{k}}{2}$ is the energy capacitance coefficient of $k$-th device. Substituting (6) into the right hand-side of (8) yields:

$$
E_{k}^{c m p}=\frac{\alpha_{k}}{2}\left(\varepsilon\left(f_{k}^{\mathrm{cmp}}\right)^{2}\left|D_{k}\right| \Phi\right)
$$

\section{Radio Frequency Uploading model}

We consider Time Division Multiple Access (TDMA) for uploading local models. We denote the uplink channel gain between the $k$-th worker and the $M$-antenna BS by $\mathbf{h}_{k} \in \mathbb{C}^{M}$. Accordingly, for a given upload interval $T_{k}^{\text {up }}$, the uplink data rate achieved by the $k$-th worker can be defined as:

$$
R_{k}^{u p}=T_{k}^{\mathrm{up}} B \log _{2}\left(1+\frac{\left|\mathbf{h}_{k}^{H} \mathbf{w}_{k}\right|^{2} P_{k}^{u p}}{\mathbf{w}_{k}^{H}\left(\sum_{k^{\prime} \neq k} \mathbf{h}_{k^{\prime}} \mathbf{h}_{k^{\prime}}^{H}+\sigma_{0}^{2} \mathbf{I}\right) \mathbf{w}_{k}}\right),
$$

where $B$ is the bandwidth, $\mathbf{w}_{k} \in \mathbb{C}^{M}$ denotes the received beamforming vectors from $M$-antenna $\mathrm{BS}, P_{k}^{u p}$ is the $k$ th worker transmit power, (.) ${ }^{H}$ stands for the Hermitian operation, $\sigma_{0}^{2}$ is the spectral density power of the additive white Gaussian noise (AWGN), and I is the identity matrix. By letting $\Gamma_{k}=B \log _{2}\left(1+\frac{\left|\mathbf{h}_{k}^{H} \mathbf{w}_{k}\right|^{2} P_{k}^{u p}}{\mathbf{w}_{k}^{H}\left(\sum_{k^{\prime} \neq k} \mathbf{h}_{k^{\prime}} \mathbf{h}_{k^{\prime}}^{H}+\sigma_{0}^{2} \mathbf{I}\right) \mathbf{w}_{k}}\right)$, the upload latency is defined as:

$$
T_{k}^{\mathrm{up}}=\frac{\xi}{\Gamma_{k}}
$$

where $\xi$ denotes the model size. Furthermore, the transmission energy consumption of the $k$-th worker is defined as:

$$
E_{k}^{\mathrm{up}}=T_{k}^{\mathrm{up}} P_{k}^{u p}
$$




\section{PROBLEM Formulation}

The aim of this work is to minimize the total energy consumed during FEEL rounds, subject to constraints on the energy consumed for computation and communication and model update and upload latencies. Particularly, to keep the model updates consistent, the selected workers should meet all required constraints. Consequently, we can formulate the optimization problem as follows:

$$
\begin{aligned}
\mathbf{P}_{1}: \min _{\substack{P_{k}^{u p}, T_{k}^{\text {up }}, T_{k}^{\text {cmp }} \\
f_{k}^{\text {cmp }}, \mathbf{w}_{k}}} & \sum_{r=1}^{R} \sum_{k}^{K} \mathbf{I}(\mathbf{k})\left(E_{k}^{c m p}+E_{k}^{\text {up }}\right) \\
\text { s.t.: } \quad & E_{k}^{\text {cmp }}+E_{k}^{\text {up }} \leq E_{k}, \quad(\forall k) \\
& T_{k}^{\text {cmp }}+T_{k}^{\text {up }}=\mathbf{T}, \quad(\forall k) \\
& P_{k}^{\min } \leq P_{k}^{u p} \leq P_{k}^{\max }, \quad(\forall k) \\
& f_{k}^{\text {min }} \leq f_{k}^{\text {cmp }} \leq f_{k}^{\max }, \quad(\forall k) \\
& R_{k}^{u p} \geq \xi, \quad(\forall k) \\
& \left|\mathbf{w}_{k}\right|^{2}=1, \quad(\forall k)
\end{aligned}
$$

where $\mathbf{I}(\mathbf{k})$ is an indicator function that specifies whether the $\mathrm{k}-t h$ worker is involved in the $r$-th round or not. Constraint (13b) ensures that the energy consumed for computation and communication does not exceed the energy budget of the $k$-th worker. The constraint $(13 \mathrm{c}$ ) is set to guarantee that the total computation and upload time is restricted to round deadline $\mathbf{T}$ to synchronize the updates. The transmit power of every selected worker is restricted in $(13 \mathrm{~d})$ to be between the minimum transmit power $P_{k}^{\mathrm{min}}$ and the maximum transmit power $P_{k}^{\max }$. Constraint $13 \mathrm{e}$ ensures that the CPU-frequency of the $k$-th worker ranges between the minimum $f_{k}^{\min }$ and maximum $f_{k}^{\max } \mathrm{CPU}$ frequencies. Constraint (13f) ensures that the upload time of the $k$-th worker is sufficient to send the model (i.e., the updated parameters) $\xi$ to the server. Last, constraint $(13 \mathrm{~g})$ ensures that the received beamforming vectors have direct direction to the M-antenna BS.

Evidently, $\mathbf{P}_{1}$ is intractable as it requires the availability of future information about the participating workers and their channels and mobility. Also, variables $P_{k}^{u p}, \mathrm{~T}_{\mathrm{k}}^{\mathrm{up}}$, and $T_{k}^{\mathrm{cmp}}$ are all coupled in constraints (13f), (13b), and (13f).

It is worth noting that the optimal beam vector $\mathbf{w}_{k}$ can be defined as [17]:

$$
\mathbf{w}_{j}^{\star}=\arg \max _{\left|\mathbf{w}_{k}\right|^{2}=1} R_{k}^{u p} \quad(\forall k) .
$$

According to Rayleight-Ritz quotient [17], [18], $\mathbf{w}_{k}^{\star}$ can be obtained by finding the eigenvector corresponding to the largest eigenvalue of the matrix $\mathbf{h}_{k} \mathbf{h}_{k}^{H}\left(\sum_{k^{\prime} \neq k} \mathbf{h}_{k^{\prime}} \mathbf{h}_{k^{\prime}}^{H}+\sigma_{0}^{2} \mathbf{I}\right)^{-1}$.

\section{Proposed ApProACH}

To solve $\mathbf{P}_{1}$, we propose a novel three-phase heuristic. In the first phase of the heuristic, a novel local training algorithm is introduced to allow workers only to include the samples that provide significant enhancement for the global model and, as a consequence, reduce the local computation energy. The details of the proposed algorithm are presented in Section $\mathrm{V}$ In the second phase, we tune the transmit time, transmit power, and local CPU speed based on the "reduced" local samples. Finally, in the third phase, we utilize the approach presented in [17] to find the optimal value of $\mathbf{w}_{k}$ which in return maximizes $R_{k}^{u p}$ to reduce the upload time and the corresponding transmit power. In our approach, all selected workers receive the global parameters from the edge server and use their entire local samples to update the received model parameters only once (i.e., initialization epoch $\varepsilon$ ) in order to specialize the global parameters and reduce the divergence between the global and local models. Then, workers use the updated model to predict the local samples and exclude the samples classified with a probability higher than a preset threshold $\vartheta$. The threshold specifies below which samples are excluded in future epochs. Given the number of local samples $\left|D_{k}\right|$, the number of excluded samples is denoted as $\iota$. Therefore, the number of samples included in future epochs is $\left|D_{k}\right|-\iota$. Accordingly, the computation time needed to complete the update task can be rewritten as:

$$
\begin{aligned}
T_{k}^{c m p}=(\varepsilon-1) & \frac{\left(\left|D_{k}\right|-\iota\right) \Phi}{f_{k}^{\mathrm{cmp}}}+\frac{\left|D_{k}\right| \Phi}{f_{k}^{\mathrm{cmp}}} \\
= & \frac{\left(\varepsilon \phi\left|D_{k}\right|\right)-\iota(\varepsilon-1)}{f_{k}^{\mathrm{cmp}}}
\end{aligned}
$$

Consequently, the corresponding total energy consumed for local computation can be defined as:

$\left.\left.E_{k}^{c m p}=\frac{\alpha_{k}}{2}(\varepsilon-1)\left(f_{k}^{\mathrm{cmp}}\right)^{2}\left(\left|D_{k}\right|-\iota\right) \Phi\right)+\frac{\alpha_{k}}{2}\left(f_{k}^{\mathrm{cmp}}\right)^{2}\left|D_{k}\right| \Phi\right)$

In Algorithm 1, the server initiates the global model parameters and determines the threshold probability that is used to select the samples to be included in the local training. The selected samples with $P \leq \vartheta$ are used to train the local model for the rest of the epochs. The steps of these algorithms are summarized in Algorithms 11 and 2. Next

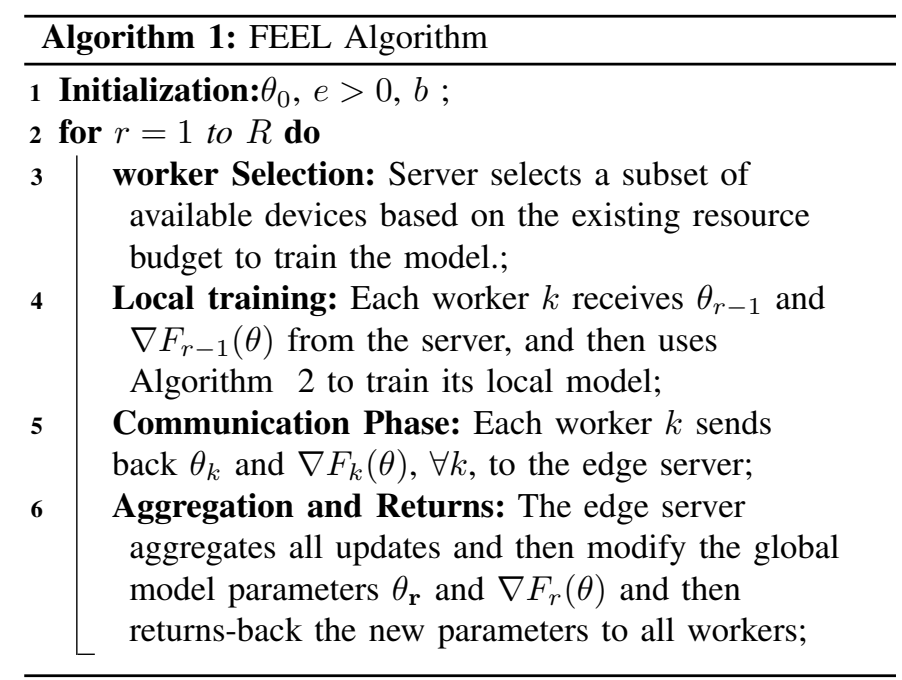

let $\rho=\left(\varepsilon \phi\left|D_{k}\right|\right)-\iota(\varepsilon-1)$, then considering (8) and (13d), 


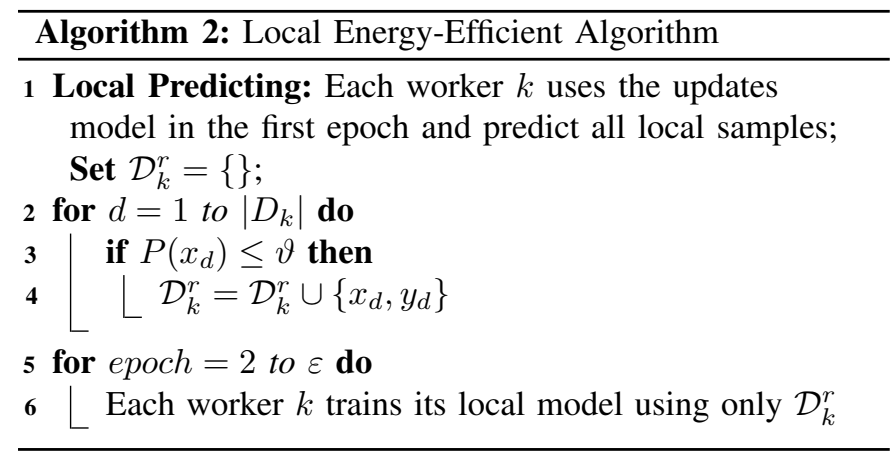

constraint 13c can be rewritten as

$$
0<\mathbf{T}-\frac{\rho}{f_{k}^{\min }} \leq T_{k}^{\mathrm{up}} \leq \mathbf{T}-\frac{\rho}{f_{k}^{\max }} \leq \mathbf{T} . \quad(\forall k)
$$

Further, let $\beta_{k}=\frac{\left|\mathbf{h}_{k}^{H} \mathbf{w}_{k}^{\star}\right|^{2}}{\mathbf{w}_{k}^{\star H}\left(\sum_{k^{\prime} \neq k} \mathbf{h}_{k^{\prime}} \mathbf{h}_{k^{\prime}}^{H}+\sigma_{0}^{2} \mathbf{I}\right) \mathbf{w}_{k}^{\star}}$. Hence from 10] and by using exponent of log rule, 13f can be derived as:

$$
P_{k}^{u p}=\frac{2^{\frac{\xi}{T_{k}^{\mathrm{up}} B}}-1}{\beta_{k}} .
$$

By substituting (18) into the right hand side of (12), we have:

$$
E_{k}^{\mathrm{up}}=T_{k}^{\mathrm{up}} \frac{2^{\frac{\xi}{T_{k}^{\mathrm{up} B}}}-1}{\beta_{k}} .
$$

$T_{k}^{\mathrm{up}}, T_{k}^{\mathrm{cmp}}$ and $f_{k}^{\mathrm{cmp}}$ should be tuned to synchronize the workers and minimize the total energy consumption of the FEEL system. From (13d), we can infer that $T_{k}^{\mathrm{up}}$ is bounded and the Golden-section search method can be used to find its optimal value [17], [19] as follows:

\begin{tabular}{|c|}
\hline If $E\left(a_{i+1}\right) \leq E\left(b_{i+1}\right)$ \\
$T_{k}^{\text {up }} \in\left[a_{i}, b_{i+1}\right]$ \\
Else \\
$T_{k}^{\text {up }} \in\left[a_{i+1}, b_{i}\right]$. \\
\hline
\end{tabular}

In view of this, $a_{i+1}=a_{i}+\varphi\left(b_{i}-a_{i}\right), b_{i+1}=a_{i}+(1-\varphi)\left(b_{i}-\right.$ $\left.a_{i}\right), \varphi=\frac{3-\sqrt{5}}{2}, a_{0}=\mathbf{T}-\frac{\rho}{f_{k}^{\min }}$, and $\left.b_{0}=\mathbf{T}-\frac{\rho}{f_{k}^{\max }} \mid 19\right]$. The optimal solution can be found by iterativly shortening the interval between the upper-bound and lower-bound using the golden ratio $\varphi$. The solution can be easily attained and the used method ensures convergence. Then, $T_{k}^{\mathrm{cmp}}$ can be calculated using (7). Finally, $f_{k}^{\mathrm{cmp}}$ is computed $13 \mathrm{e}$ :

$$
f_{k}^{\mathrm{cmp}}=\frac{\left(\varepsilon \phi\left|D_{k}\right|\right)-\iota(\varepsilon-1)}{T_{k}^{\mathrm{cmp}}}
$$

\section{Simulation AND Numerical RESUlts}

In our experiments, we consider a FEEL environment as in 1 with a total bandwidth of $B=1 \mathrm{MHz}$, and noise power $\sigma^{2}=10^{-6}$. The distance between the edge workers and the BS is uniformly distributed between $5 \mathrm{~m}$ and $20 \mathrm{~m}$. For the wireless channel model, we use Rician distribution with a Rician factor of $8 \mathrm{~dB}$ and a path loss exponent factor of 3.2. The number of antennas is $m=8$ for the BS and $m=1$ for each of the $k$ workers. The maximum and minimum transmit powers are set

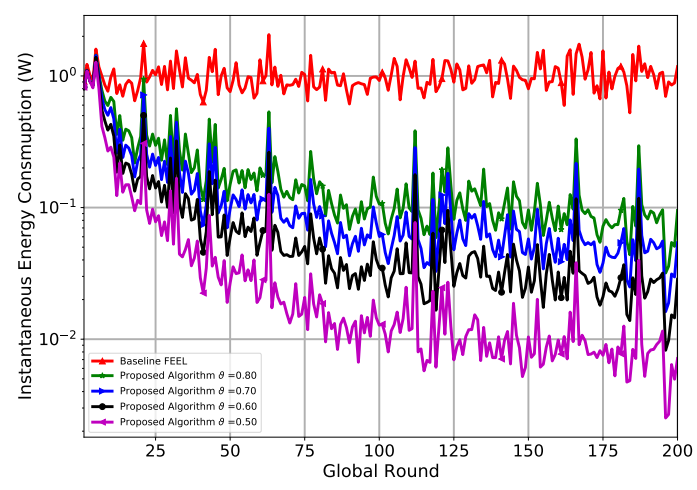

Fig. 2: Instantaneous Energy Consumption when the number of global rounds is 200 .

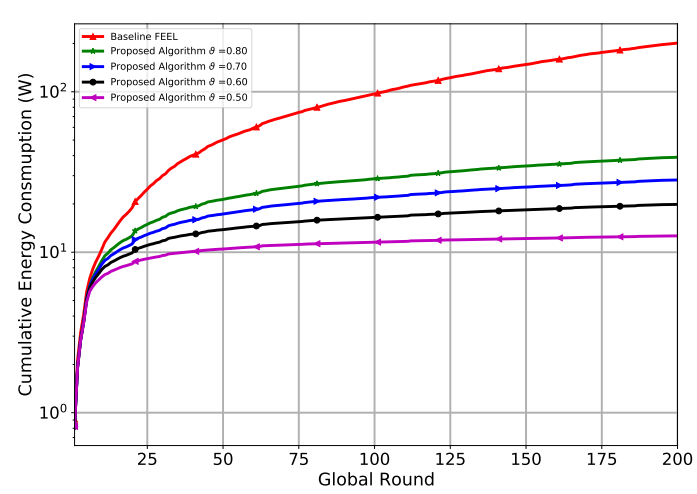

Fig. 3: Cumulative Energy Consumption when the number of global rounds is 200 .

to $P_{\max }=20 \mathrm{dBm}$ and $P_{\text {min }}=-10 \mathrm{dBm}$, respectively. We use the MNIST datasets under a realistic federated setting, imbalanced and non-i.i.d data distribution with 1000 users, and different convolutional neural networks (CNN) models. We utilize the mini-batch SGD as a local solver and evaluate the global model every round. The data is split into $80 \%$ for training and $20 \%$ for testing.

Figs. 2 and 3 show the energy consumption during FEEL rounds when the number of global rounds is 200 . It is evident that the proposed algorithm substantially reduces the total consumed energy. This stems from excluding the samples having less impact on the model training, affecting computation and communication energy consumption. For computation, only a subset of the workers' samples is injected into training for $(\varepsilon-1)$ epochs. In contrast, the baseline FEEL algorithm consumes more energy as all local samples are included in $\varepsilon$ local iterations, which affects the transmission power.

Figs. 4 and 5 show the identification accuracy and loss of handwritten digits (MNIST) when the number of global rounds is 200 and $\vartheta=0.5,0.6,0.7$, and 0.8 . From these figures, it is evident that the proposed algorithm provides approximately similar accuracy and loss, especially when the threshold probability is higher than 0.70 . However, both accuracy and loss worsen when the threshold probability is lower than 0.70 as we can see when $\vartheta=0.50$. This is because most of the excluded samples are predicted with 


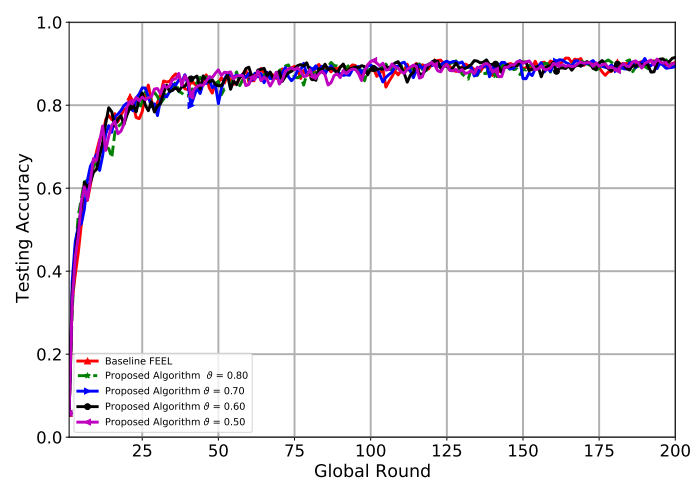

Fig. 4: Testing Accuracy (MNIST) when the number of global rounds is 200 .

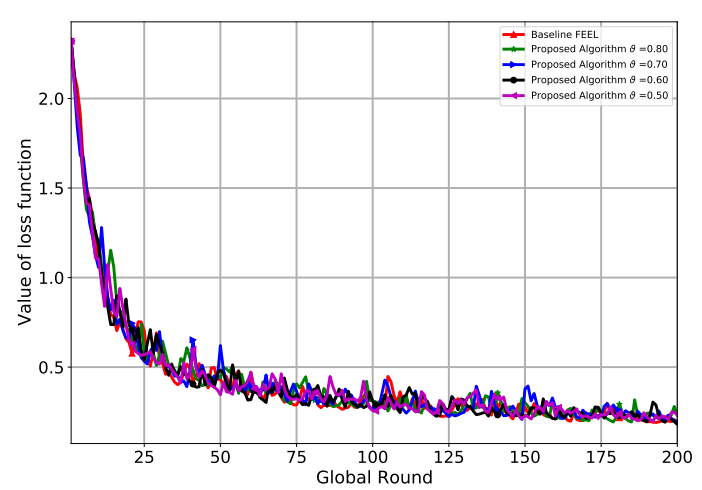

Fig. 5: Value of the loss function (MNIST) when the number of global rounds is 200 .

low probability, decreasing the number of samples used to train the local models for the rest of the epochs. Overall, our proposed approach considers computation and communications aspects that lead to significant energy efficiency enhancements supporting synchronized FEEL systems' reallife deployments. The observed enhancements stem from our proposed methods to intelligently exclude training samples that do not significantly contribute to the global model.

\section{CONCLUSION}

In this work, a novel energy-efficient FEEL approach was proposed. Our proposed approach takes advantage of local computation and communication resources to substantially reduce the energy consumed by selected workers to train their local models. In our proposed approach, each worker tunes the received global model parameters while intelligently excluding the predicted samples with high probability based on a predefined threshold as such samples do not significantly contribute to the learning model and can adversely impact energy consumption. The proposed approach tunes the transmit power and local CPU speed of workers in a FEEL system to enhance energy efficiency. Our experimental results demonstrate outstanding potential for reducing the total energy consumption of FEEL systems. Eventually, we show that energy consumption can be reduced by optimizing the available computation and communication resources and designing efficient local training algorithms. For future work, investigating the threshold's optimal value should be considered for a more efficient local training algorithm.

\section{ACKNOWLEDGEMENT}

This publication was made possible by NPRP-Standard (NPRP-S) Thirteen (13th) Cycle grant \# NPRP13S-0201200219 from the Qatar National Research Fund (a member of Qatar Foundation). The findings herein reflect the work, and are solely the responsibility, of the authors.

\section{REFERENCES}

[1] G. M. D. T. Forecast, "Cisco visual networking index: global mobile data traffic forecast update, 2017-2022," Update, vol. 2017, p. 2022, 2019.

[2] Y. LeCun, Y. Bengio, and G. Hinton, "Deep learning," nature, vol. 521, no. 7553 , p. 436,2015

[3] G. Zhu, D. Liu, Y. Du, C. You, J. Zhang, and K. Huang, "Toward an intelligent edge: wireless communication meets machine learning," IEEE Communications Magazine, vol. 58, no. 1, pp. 19-25, 2020.

[4] K. B. Letaief, W. Chen, Y. Shi, J. Zhang, and Y.-J. A. Zhang, "The roadmap to 6g: Ai empowered wireless networks," IEEE Communications Magazine, vol. 57, no. 8, pp. 84-90, 2019.

[5] X. Mo and J. Xu, "Energy-efficient federated edge learning with joint communication and computation design," arXiv preprint arXiv:2003.00199, 2020.

[6] T. Li, A. K. Sahu, A. Talwalkar, and V. Smith, "Federated learning: Challenges, methods, and future directions," IEEE Signal Processing Magazine, vol. 37, no. 3, pp. 50-60, 2020.

[7] F. Sattler, S. Wiedemann, K.-R. Müller, and W. Samek, "Robust and communication-efficient federated learning from non-iid data," IEEE transactions on neural networks and learning systems, vol. 31, no. 9, pp. 3400-3413, 2019.

[8] Y. Lin, S. Han, H. Mao, Y. Wang, and W. J. Dally, "Deep gradient compression: Reducing the communication bandwidth for distributed training," arXiv preprint arXiv:1712.01887, 2017.

[9] S. Wang, T. Tuor, T. Salonidis, K. K. Leung, C. Makaya, T. He, and K. Chan, "Adaptive federated learning in resource constrained edge computing systems," IEEE Journal on Selected Areas in Communications, vol. 37, no. 6, pp. 1205-1221, 2019.

[10] G. Zhu, Y. Wang, and K. Huang, "Broadband analog aggregation for low-latency federated edge learning," IEEE Transactions on Wireless Communications, vol. 19, no. 1, pp. 491-506, 2019.

[11] T. Nishio and R. Yonetani, "Client selection for federated learning with heterogeneous resources in mobile edge," in ICC 2019-2019 IEEE International Conference on Communications (ICC), pp. 1-7. IEEE, 2019.

[12] K. Yang, T. Jiang, Y. Shi, and Z. Ding, "Federated learning via over-theair computation," [Online]. Available: https://arxiv.org/abs/1812.11750, 2018.

[13] L. Liu, J. Zhang, S. Song, and K. B. Letaief, "Edge-assisted hierarchical federated learning with non-iid data," arXiv preprint arXiv:1905.06641 2019.

[14] G. Zhu, Y. Du, D. Gunduz, and K. Huang, "One-bit over-the-air aggregation for communication-efficient federated edge learning: Design and convergence analysis," arXiv preprint arXiv:2001.05713, 2020.

[15] Q. Zeng, Y. Du, K. Huang, and K. K. Leung, "Energy-efficient radio resource allocation for federated edge learning," in 2020 IEEE International Conference on Communications Workshops (ICC Workshops), pp 1-6. IEEE, 2020.

[16] S. Wang, M. Chen, W. Saad, and C. Yin, "Federated learning for energyefficient task computing in wireless networks," in ICC 2020-2020 IEEE International Conference on Communications (ICC), pp. 1-6. IEEE, 2020.

[17] H.-V. Tran, G. Kaddoum, H. Elgala, C. Abou-Rjeily, and H. Kaushal, "Lightwave power transfer for federated learning-based wireless networks," IEEE Communications Letters, vol. 24, no. 7, 2020.

[18] B. N. Parlett, The symmetric eigenvalue problem. NJ, USA: PrenticeHall, Inc. Upper Saddle River, 1998.

[19] W. H. Press, S. A. Teukolsky, W. T. Vetterling, and B. P. Flannery, Numerical Recipes 3rd Edition: The Art of Scientific Computing. New York: Cambridge University Press, 2007. 